\title{
Linear Barycentric Rational Method for Two-Point Boundary Value Equations
}

\author{
Qian Ge $\mathbb{D}^{1,2}$ and Xiaoping Zhang $\mathbb{D}^{2}$ \\ ${ }^{1}$ School of Mathematics and Statistics, Shandong Normal University, Jinan 250358, China \\ ${ }^{2}$ School of Science, Shandong Jianzhu University, Jinan 250101, China \\ Correspondence should be addressed to Xiaoping Zhang; geqian@sdjzu.edu.cn
}

Received 22 September 2020; Revised 22 October 2020; Accepted 24 March 2021; Published 13 April 2021

Academic Editor: Ljubisa Kocinac

Copyright ( 2021 Qian Ge and Xiaoping Zhang. This is an open access article distributed under the Creative Commons Attribution License, which permits unrestricted use, distribution, and reproduction in any medium, provided the original work is properly cited.

Linear barycentric rational method for solving two-point boundary value equations is presented. The matrix form of the collocation method is also obtained. With the help of the convergence rate of the interpolation, the convergence rate of linear barycentric rational collocation method for solving two-point boundary value problems is proved. Several numerical examples are provided to validate the theoretical analysis.

\section{Introduction}

The analysis of many physical phenomena and engineering problems can be reduced to solving the boundary value problem of differential equation, most of which need to be solved by the numerical method. The barycentric interpolation method is a high precision calculation method, and a strong form of collocation that relies on differential equation, which has been studied extensively by many scholars. The linear barycentric rational method (LBRM) [1-3] has been used to solve certain problems such as delay Volterra integro-differential equations [4], Volterra integral equations [5-7], biharmonic equation [8], beam force vibration equation [9], boundary value problems [10], heat conduction problems [11], plane elastic problems [12], incompressible plane elastic problems [13], nonlinear problems [14], and so on $[1,15]$.

In this article, we pay our attention to the numerical solution of two-point boundary value problems:

$$
\begin{aligned}
(\mathrm{Tu})(x) & :=u^{\prime \prime}(x)+\mathrm{qu}(x)=f(x), \quad x \in(a, b), \\
u(a) & =u_{\ell}, u(b)=u_{r} .
\end{aligned}
$$

Let the interval $[a, b]$ be partitioned into $n$ uniform part with $h=(b-a) / n$ and $x_{0}, x_{1}, \ldots, x_{n}$ with its related function $f\left(x_{i}\right), i=0,1, \ldots, n$. For any $0 \leq d \leq n$, with
$P\left(x_{i}\right), i=0,1, \ldots, n-d$, to be the interpolation function at the point $x_{i}, x_{i+1}, \ldots, x_{i+d}$, then we have $P_{i}\left(x_{k}\right)=$ $f\left(x_{k}\right), k=i, i+1, \ldots, i+d$, and

$$
r(x)=\frac{\sum_{i=0}^{n-d} \lambda_{i}(x) P_{i}(x)}{\sum_{i=0}^{n-d} \lambda_{i}(x)}
$$

where

$$
\lambda_{i}(x)=\frac{(-1)^{i}}{\left(x-x_{i}\right) \cdots\left(x-x_{i+d}\right)} .
$$

Change the polynomial $P_{i}(x)$ into the Lagrange interpolation form as

$$
P_{i}(x)=\sum_{k=i}^{i+d} \prod_{j=i, j \neq k}^{i+d} \frac{x-x_{j}}{x_{k}-x_{j}} f_{k}
$$

Combining (7) and (5) together, we get

$$
\sum_{i=0}^{n-d} \lambda_{i}(x) P_{i}(x)=\sum_{i=0}^{n-d}(-1)^{i} \sum_{k=i}^{i+d} \frac{1}{x-x_{k}} \prod_{i, j \neq k}^{i+d} \frac{1}{x_{k}-x_{j}} f_{k}=\sum_{k=0}^{n} \frac{w_{k}}{x-x_{k}} f_{k},
$$

where $w_{k}=\sum_{i \in J_{k}}(-1)^{i} \prod_{j=i, j \neq k}^{i+d} 1 /\left(x_{k}-x_{j}\right) \quad$ and $\quad J_{k}=\{i \in$ $I ; k-d \leq i \leq k\}$. 
Then we get

$$
r(x)=\frac{\sum_{j=0}^{n}\left[w_{j} /\left(x-x_{j}\right)\right] f_{j}}{\sum_{j=0}^{n} w_{j} /\left(x-x_{j}\right)},
$$

where its basis function is

$$
L_{j}(x)=\frac{w_{j} /\left(x-x_{j}\right)}{\sum_{k=0}^{n} w_{k} /\left(x-x_{k}\right)} .
$$

For the equidistant point, its weight function is

$$
w_{j}=(-1)^{n-j} C_{n}^{j} \text {. }
$$

The Chebyshev point of the second kind is

$$
x_{j}=\cos \frac{j \pi}{n}, j=0,1, \ldots, n,
$$

and its weight function is

$$
w_{j}=(-1)^{j} \delta_{j}, \delta_{j}= \begin{cases}\frac{1}{2}, & j=0, n, \\ 1, & \text { ortherwise. }\end{cases}
$$

Consider the barycentric interpolation function as

$$
u_{n}(x)=\sum_{j=0}^{n} L_{j}(x) u_{j}
$$

and the numerical scheme is given as

$$
\sum_{j=0}^{n} u_{j} L_{j}^{\prime \prime}(x)+q \sum_{j=0}^{n} u_{j} L_{j}(x)=f(x) .
$$

By using the notation of the differential matrix, equation (13) is denoted as matrices in the form of

$$
\sum_{j=0}^{n} D_{i j}^{(2)} u_{j}+q \sum_{j=0}^{n} \delta_{i j} u_{j}=f\left(x_{i}\right)
$$

where $i=1,2, \ldots, n$.

Equation (13) is written as matrices in the form of

$$
\left[\mathbf{D}^{(2)}+\mathbf{q I}\right] \mathbf{u}=\mathbf{f},
$$

where $\quad \mathbf{L}:=\mathbf{D}^{(2)}+\mathbf{q} \mathbf{I}, \mathbf{u}=\left[u_{0}, u_{1}, u_{2}, \ldots, u_{n}\right]^{\mathrm{T}}, \mathbf{D}^{(k)}=$ $\left[D_{i j}^{(k)}\right]_{(n+1) \times(n+1)}$,

$$
\begin{aligned}
& D_{i j}^{(1)}= \begin{cases}\frac{\omega_{j} / \omega_{i}}{x_{i}-x_{j}}, & i \neq j, \\
-\sum_{k \neq i} D_{i k}^{(1)}, & i=j,\end{cases} \\
& D_{i j}^{(2)}= \begin{cases}2 D_{i j}^{(1)}\left(D_{i i}^{(1)}-\frac{1}{x_{i}-x_{j}}\right), & i \neq j, \\
-\sum_{k \neq i} D_{i k}^{(2)}, & i=j,\end{cases}
\end{aligned}
$$

and $\quad \mathbf{q}=\operatorname{diag}[q], f=\left[f\left(x_{0}\right), f\left(x_{1}\right), f\left(x_{2}\right), \ldots, f\left(x_{n}\right)\right]^{\mathrm{T}}$. Using interpolation formulas, boundary conditions can be discretized into

$$
\sum_{j=0}^{n} D_{1 j}^{(1)} u_{j}=a, \sum_{j=0}^{n} D_{n j}^{(1)} u_{j}=b .
$$

\section{Convergence and Error Analysis}

With the error function of difference formula

$$
e(x):=u(x)-r(x)=\left(x-x_{i}\right) \cdots\left(x-x_{i+d}\right)\left[x_{i}, x_{i+1}, \ldots, x_{i+d}, x\right] f,
$$

and

$$
e(x)=\frac{\sum_{i=0}^{n-d} \lambda_{i}(x)\left(u(x)-P_{i}(x)\right)}{\sum_{i=0}^{n-d} \lambda_{i}(x)}=\frac{A(x)}{B(x)}=O\left(h^{d+1}\right),
$$

where $A(x):=\sum_{i=0}^{n-d}(-1)^{i}\left[x_{i}, \ldots, x_{i+d}, x\right] f, B(x):=\sum_{i=0}^{n-d} \lambda_{i}$ $(x)$. Taking the numerical scheme

$$
\sum_{j=0}^{n} y_{j} L_{j}^{\prime \prime}(x)+q \sum_{j=0}^{n} y_{j} L_{j}(x)=f(x) .
$$

Combining (20) and (1), we have

$$
\operatorname{Te}(x):=e^{\prime \prime}(x)+\mathrm{qe}(x)=R_{f}(x),
$$

where $R_{f}(x)=f(x)-f\left(x_{k}\right), k=0,1,2, \ldots, n$.

The following Lemma has been proved by Jean-Paul Berrut in [13].

Lemma 1 (see [13]). For $e(x)$ defined in (18), we have

$$
\begin{cases}|e(x)| \leq C h^{d+1}, & u \in C^{d+2}[a, b], \\ \left|e^{\prime}(x)\right| \leq C h^{d}, & u \in C^{d+3}[a, b], \\ \left|e^{\prime \prime}(x)\right| \leq C h^{d-1}, & u \in C^{d+4}[a, b], d \geq 1 .\end{cases}
$$

Let $u(x)$ be the solution of $(1)$ and $u_{n}(x)$ is the numerical solution, then we have

$$
T u_{n}\left(x_{k}\right)=f\left(x_{k}\right), \quad k=0,1,2, \ldots, n,
$$

and

$$
\lim _{n \longrightarrow \infty} u_{n}(x)=u(x)
$$

The results can be obtained in the reference of [14].

Based on the above lemma, we derive the following theorem.

Theorem 1. Let $u_{n}(x): T u_{n}(x)=f(x), u_{n}^{*}(x): T u_{n}^{*}(x)=$ $f^{*}(x)$, and $f(x) \in C[a, b]$, we have

$$
\left|u_{n}(x)-u_{n}^{*}(x)\right| \leq C h^{d-1}
$$


Proof. As $\mathbf{L}:=\mathbf{D}^{(2)}+\mathbf{Q I}$, where

$$
\mathbf{D}^{2}=\left[\begin{array}{cccccc}
D_{00}^{(2)} & D_{01}^{(2)} & D_{02}^{(2)} & D_{03}^{(2)} & \cdots & D_{0 n}^{(2)} \\
D_{10}^{(2)} & D_{11}^{(2)} & D_{12}^{(2)} & D_{13}^{(2)} & \cdots & D_{1 n}^{(2)} \\
D_{20}^{(2)} & D_{21}^{(2)} & D_{22}^{(2)} & D_{23}^{(2)} & \cdots & D_{2 n}^{(2)} \\
D_{30}^{(2)} & D_{31}^{(2)} & D_{32}^{(2)} & D_{33}^{(2)} & \cdots & D_{3 n}^{(2)} \\
\cdots & \cdots & \cdots & \cdots & \cdots & \cdots \\
D_{n-1,0}^{(2)} & D_{n-1,1}^{(2)} & D_{n-1,2}^{(2)} & D_{n-2,3}^{(2)} & \cdots & D_{n-1, n}^{(2)} \\
D_{n 0}^{(2)} & D_{n 1}^{(2)} & D_{n 2}^{(2)} & D_{n 3}^{(2)} & \cdots & D_{n n}^{(2)}
\end{array}\right],
$$

and

$$
\mathbf{L}:=\mathbf{D}^{2}+\mathbf{Q I}=\left[\begin{array}{cccccc}
q+D_{00}^{(2)} & D_{01}^{(2)} & D_{02}^{(2)} & D_{03}^{(2)} & \cdots & D_{0 n}^{(2)} \\
D_{10}^{(2)} & q+D_{11}^{(2)} & D_{12}^{(2)} & D_{13}^{(2)} & \cdots & D_{1 n}^{(2)} \\
D_{20}^{(2)} & D_{21}^{(2)} & q+D_{22}^{(2)} & D_{23}^{(2)} & \cdots & D_{2 n}^{(2)} \\
D_{30}^{(2)} & D_{31}^{(2)} & D_{32}^{(2)} & D_{33}^{(2)} & \cdots & D_{3 n}^{(2)} \\
\cdots & \cdots & \cdots & \cdots & \cdots & \cdots \\
D_{n-1,0}^{(2)} & D_{n-1,1}^{(2)} & D_{n-1,2}^{(2)} & D_{n-2,3}^{(2)} & \cdots & D_{n-1, n}^{(2)} \\
D_{n 0}^{(2)} & D_{n 1}^{(2)} & D_{n 2}^{(2)} & D_{n 3}^{(2)} & \cdots & q+D_{n n}^{(2)}
\end{array}\right] .
$$

Putting column 2, column 3, column $n$ added to column 1, we have

$$
\mathbf{D}^{2}=\left[\begin{array}{cccccc}
0 & D_{01}^{(2)} & D_{02}^{(2)} & D_{03}^{(2)} & \cdots & D_{0 n}^{(2)} \\
0 & D_{11}^{(2)} & D_{12}^{(2)} & D_{13}^{(2)} & \cdots & D_{1 n}^{(2)} \\
0 & D_{21}^{(2)} & D_{22}^{(2)} & D_{23}^{(2)} & \cdots & D_{2 n}^{(2)} \\
0 & D_{31}^{(2)} & D_{32}^{(2)} & D_{33}^{(2)} & \cdots & D_{3 n}^{(2)} \\
0 & \cdots & \cdots & \cdots & \cdots & \cdots \\
0 & D_{n-1,1}^{(2)} & D_{n-1,2}^{(2)} & D_{n-2,3}^{(2)} & \cdots & D_{n-1, n}^{(2)} \\
0 & D_{n 1}^{(2)} & D_{n 2}^{(2)} & D_{n 3}^{(2)} & \cdots & D_{n n}^{(2)}
\end{array}\right],
$$

which means the matrix $\mathbf{D}^{(2)}$ is the singular matrix.

Similarly we have

$$
\mathbf{L}=\left[\begin{array}{cccccc}
q & D_{01}^{(2)} & D_{02}^{(2)} & D_{03}^{(2)} & \cdots & D_{0 n}^{(2)} \\
q & q+D_{11}^{(2)} & D_{12}^{(2)} & D_{13}^{(2)} & \cdots & D_{1 n}^{(2)} \\
q & D_{21}^{(2)} & q+D_{22}^{(2)} & D_{23}^{(2)} & \cdots & D_{2 n}^{(2)} \\
q & D_{31}^{(2)} & D_{32}^{(2)} & q+D_{33}^{(2)} & \cdots & D_{3 n}^{(2)} \\
q & \cdots & \cdots & \cdots & \cdots & \cdots \\
q & D_{n-1,1}^{(2)} & D_{n-1,2}^{(2)} & D_{n-2,3}^{(2)} & \cdots & D_{n-1, n}^{(2)} \\
q & D_{n 1}^{(2)} & D_{n 2}^{(2)} & D_{n 3}^{(2)} & \cdots & q+D_{n n}^{(2)}
\end{array}\right],
$$

and then we assume $|\mathbf{L}| \neq 0$ with $q \neq 0$, $u_{n}(x)=\sum_{j=0}^{n} L_{j}(x) f_{j}, u_{n}^{*}(x)=\sum_{j=0}^{n} L_{j}(x) f_{j}^{*}, \quad$ where. $U_{n}=\left(f\left(x_{0}\right), f\left(x_{1}\right), \ldots, f\left(x_{n}\right)\right)^{T}$ and $U_{n}^{*}=\left(f^{*}\left(x_{0}\right), f^{*}\left(x_{1}\right), \ldots, f^{*}\left(x_{n}\right)\right)^{T}$.
By

$$
U_{n}-U_{n}^{*}=\mathbf{L}^{-1}\left(\mathbf{L} U_{n}-F_{n}^{*}\right),
$$

which means

$$
u_{n}(x)-u_{n}^{*}(x)=\sum_{j=0}^{n} M_{j}(x) \operatorname{Te}(x),
$$

where $M_{j}(x)$ is the element of matrix $\mathbf{L}^{-1}$.

Then we have

$$
\left|u_{n}(x)-u_{n}^{*}(x)\right| \leq\left|\sum_{j=0}^{n} M_{j}(x)\right||\operatorname{Te}(x)| \leq \mathrm{Ch}^{d-1} .
$$

The proof is completed.

We know that the central difference method can achieve quadratic convergence and the convergence order is the same as that of $d=3$. When $d>3$, the convergence of the barycentric rational method is better than that of the central difference method.

\section{Numerical Example}

Example 1. Consider the two-point boundary value:

$$
-y^{\prime \prime}+400 y=-400 \cos ^{2} \pi x-2 \pi^{2} \cos 2 \pi x,
$$

$$
y(0)=y(1)=0,
$$

and its analysis solution is

$$
y(x)=\frac{e^{-20}}{1+e^{-20}} e^{20 x}+\frac{1}{1+e^{-20}} e^{-20 x}-\cos ^{2} \pi x .
$$

In this example, we consider the two-point boundary value equations with the boundary condition $y(0)=y(1)=0$. In Table 1 , the convergence rate of equidistant nodes with different $d$ is $O\left(h^{d}\right)$; in Table 2, the convergence rate of the Chebyshev point of the second kind with different $d$ is $O\left(h^{d+2}\right), d \geq 2$. From Theorem 1, the convergence rate is $O\left(h^{d-1}\right)$, and there are no convergence rates as $d=1$. Here the convergence rate is $O(h)$ and $O\left(h^{2}\right)$ in Tables 1 and 2 for $d=1$, respectively, and we will give exact analysis in other paper.

Example 2. Consider the two-point boundary value.

$$
\begin{aligned}
y^{\prime \prime}+y^{\prime} \sin x+y e^{x}= & -16 \pi^{2} \sin 4 \pi x+4 \pi \sin x \cos 4 \pi x \\
& +e^{x}(2+\sin 4 \pi x), \quad-1<x<1,
\end{aligned}
$$

with the boundary condition

$$
y(-1)+y^{\prime}(-1)=2+4 \pi, y(1)+y^{\prime}(1)=2+4 \pi,
$$

and its analysis solution is

$$
y(x)=2+\sin 4 \pi x .
$$

In this example, we consider the variable coefficient of two-point boundary value equations with the boundary 
TABLE 1: Errors of the equidistant nodes with different $d$.

\begin{tabular}{lccccc}
\hline$n$ & $d=1$ & $d=2$ & $d=3$ & $d=4$ \\
\hline 10 & $5.6796 e-02$ & $4.7174 e-02$ & $3.6770 e-02$ & $2.9692 e-02$ & $2.5240 e-02$ \\
20 & $3.1866 e-02$ & $2.0707 e-02$ & $1.2295 e-02$ & $7.5473 e-03$ & $4.6949 e-03$ \\
40 & $1.1899 e-02$ & $4.8530 e-03$ & $1.8024 e-03$ & $6.8958 e-04$ & $2.6519 e-04$ \\
80 & $4.0465 e-03$ & $8.2037 e-04$ & $1.7027 e-04$ & $3.6416 e-05$ & $7.8060 e-06$ \\
160 & $1.4721 e-03$ & $1.1821 e-04$ & $1.2993 e-05$ & $1.4706 e-06$ & $1.6657 e-07$ \\
320 & $4.9182 e-04$ & $1.5796 e-05$ & $8.9500 e-07$ & $5.2131 e-08$ & $3.0430 e-09$ \\
640 & $1.5719 e-04$ & $2.0375 e-06$ & $5.8674 e-08$ & $1.7409 e-09$ & $5.1202 e-11$ \\
\hline
\end{tabular}

TABle 2: Errors of the Chebyshev point with different $d$.

\begin{tabular}{|c|c|c|c|c|c|}
\hline$n$ & $d=1$ & $d=2$ & $d=3$ & $d=4$ & $d=5$ \\
\hline 10 & $4.7235 e-02$ & $3.4143 e-02$ & $2.3198 e-02$ & $1.0658 e-02$ & $4.2299 e-03$ \\
\hline 20 & $2.0966 e-02$ & $4.9675 e-03$ & $2.3057 e-03$ & $1.5490 e-03$ & $1.0185 e-03$ \\
\hline 40 & $5.0812 e-03$ & $3.3376 e-04$ & $7.5530 e-05$ & $3.1018 e-05$ & $1.2517 e-05$ \\
\hline 80 & $1.2482 e-03$ & $2.0099 e-05$ & $2.1222 e-06$ & $4.5138 e-07$ & $9.5181 e-08$ \\
\hline 160 & $2.9995 e-04$ & $1.2091 e-06$ & $6.1512 e-08$ & $6.5988 e-09$ & $6.9630 e-10$ \\
\hline 320 & $7.3360 e-05$ & $7.3634 e-08$ & $1.8414 e-09$ & $1.0610 e-10$ & $2.8788 e-10$ \\
\hline 640 & $1.8092 e-05$ & $4.5328 e-09$ & $5.7970 e-11$ & $1.5463 e-10$ & $6.0430 e-09$ \\
\hline
\end{tabular}

TABLE 3: Errors of the equidistant nodes with different $d$.

\begin{tabular}{lccccc}
\hline$n$ & $d=1$ & $d=2$ & $d=3$ & $d=4$ & $d=5$ \\
\hline 10 & $3.1903 e+00$ & $5.8183 e+00$ & $4.2584 e+00$ & $3.3515 e+00$ & $3.8150 e+00$ \\
20 & $9.0854 e-01$ & $1.8487 e-01$ & $4.7472 e-02$ & $1.9234 e-04$ & $2.1227 e-02$ \\
40 & $1.9690 e-01$ & $3.8711 e-02$ & $5.9273 e-04$ & $2.3577 e-06$ & $4.9300 e-05$ \\
80 & $4.4235 e-02$ & $3.9481 e-03$ & $4.9399 e-06$ & $1.9939 e-08$ & $2.4228 e-07$ \\
160 & $9.6887 e-03$ & $3.4240 e-04$ & $1.9523 e-07$ & $1.6331 e-09$ & $2.8181 e-09$ \\
320 & $2.0060 e-03$ & $2.7549 e-05$ & $5.5624 e-09$ & $2.1374 e-08$ & $7.9052 e-08$ \\
640 & $3.7877 e-04$ & $2.1177 e-06$ & $1.0976 e-09$ & & $1.2137 e-06$ \\
\hline
\end{tabular}

TABLE 4: Errors of the Chebyshev point with different $d$.

\begin{tabular}{lccccc}
\hline$n$ & $d=1$ & $d=2$ & $d=3$ & $d=4$ & $d=5$ \\
\hline 10 & $1.3687 e+01$ & $1.0866 e+02$ & $2.9315 e+02$ & $6.7980 e+02$ & $2.2744 e+00$ \\
20 & $2.9375 e+00$ & $2.2412 e+01$ & $2.0846 e+01$ & $1.3524 e+00$ & $3.1001 e+01$ \\
40 & $1.0678 e+00$ & $4.9833 e+00$ & $9.5860 e-01$ & $9.3346 e-02$ & $7.5788 e-01$ \\
80 & $4.4216 e-01$ & $1.1571 e+00$ & $2.6706 e-02$ & $5.6811 e-03$ & $8.5273 e-03$ \\
160 & $1.9821 e-01$ & $2.7788 e-01$ & $1.7104 e-03$ & $3.4460 e-04$ & $2.5039 e-05$ \\
320 & $9.3181 e-02$ & $6.8032 e-02$ & $4.9655 e-04$ & $2.1132 e-05$ & $1.3480 e-07$ \\
640 & $4.5032 e-02$ & $1.6827 e-02$ & $7.8365 e-05$ & & \\
\hline
\end{tabular}

condition $y(-1)+y^{\prime}(-1)=2+4 \pi, y(1)+y^{\prime}(1)=2+4 \pi$. In Table 3 , the convergence rate of equidistant nodes with different $d$ is $O\left(h^{d}\right)$; in Table 4, the convergence rate of the Chebyshev point of second kind with different $d$ is $O\left(h^{d+2}\right), d \geq 2$.

\section{Concluding Remarks}

In this paper, the numerical approximation of linear barycentric rational collocation method for solving two-point boundary value equations is presented. The matrix form of the algorithm is given for the simple calculation; with the help of Newton formula, the error function of the convergence rate $O\left(h^{d-1}\right)$ is also obtained. For the constant coefficient and variable coefficient of two-point boundary value equations, numerical results show that the convergence rate can reach $O\left(h^{d}\right)$ for the equidistant nodes and $O\left(h^{d+2}\right)$ for the Chebyshev point of the second kind with $d \geq 2$. For the special case of $d=1$, there are still convergence rates with $O(h)$, and the analysis of this phenomenon will be presented in other papers.

\section{Data Availability}

The data that support the findings of this study are available from the corresponding author upon reasonable request. 


\section{Conflicts of Interest}

The authors declare that they have no conflicts of interest.

\section{Acknowledgments}

The support from the Nature Science Foundation of Shandong (No. ZR2019PA021) is gratefully acknowledged.

\section{References}

[1] M. S. Floater and K. Hormann, "Barycentric rational interpolation with no poles and high rates of approximation," Numerische Mathematik, vol. 107, no. 2, pp. 315-331, 2007.

[2] G. Klein and J.-P. Berrut, "Linear rational finite differences from derivatives of barycentric rational interpolants," SIAM Journal on Numerical Analysis, vol. 50, no. 2, pp. 643-656, 2012.

[3] G. Klein and J.-P. Berrut, "Linear barycentric rational quadrature," BIT Numerical Mathematics, vol. 52, no. 2, pp. 407-424, 2012.

[4] A. Abdi and J.-P. Berrut, A. H. Seyyed, "The linear barycentric rational method for a class of delay volterra integro-differential equations," Journal of Scientific Computing, vol. 75, no. 3, pp. 1757-1775, 2018.

[5] J. P. Berrut, S. A. Hosseini, and G. Klein, "The linear barycentric rational quadrature method for Volterra integral equations," SIAM Journal on Scientific Computing, vol. 36, no. 1, pp. 105-123, 2014.

[6] J. Li and Y. L. Cheng, "Linear barycentric rational collocation method for solving second-order Volterra integro-differential equation," Computational and Applied Mathematics, vol. 92, 2020.

[7] M. Li and C. Huang, "The linear barycentric rational quadrature method for auto-convolution Volterra integral equations," Journal of Scientific Computing, vol. 78, no. 1, pp. 549-564, 2019.

[8] J.-Y. Greengard and L. Greengardz, "A fast adaptive numerical method for stiff two-point boundary value problems," SIAM Journal on Scientific Computing, vol. 18, no. 2, pp. 403-429, 1997.

[9] J. Li and Y. L. Cheng, "Linear barycentric rational collocation method for solving heat conduction equation," Numerical Methods for Partial Differential Equations, vol. 37, no. 1, pp. 533-545, 2020.

[10] Z. Wang, L. Zhang, Z. Xu, and J. Li, "Barycentric interpolation collocation method based on mixed displacement-stress formulation for solving plane elastic problems," Chinese Journal of Applied Mechanics, vol. 35, no. 2, pp. 304-309, 2018.

[11] Z. Wang, Z. Xu, and J. Li, "Mixed barycentric interpolation collocation method of displacement-pressure for incompressible plane elastic problems," Chinese Journal of Applied Mechanics, vol. 35, no. 3, pp. 195-201, 2018.

[12] Z. Wang and S. Li, Barycentric Interpolation Collocation Method for Nonlinear Problems, National Defense Industry Press, Beijing, China, 2015.

[13] E. Cirillo and K. Hormann, "On the Lebesgue constant of barycentric rational Hermite interpolants at equidistant nodes," Journal of Computational and Applied Mathematics, vol. 349, pp. 292-301, 2019.

[14] J.-P. Berrut, M. S. Floater, and G. Klein, "Convergence rates of derivatives of a family of barycentric rational interpolants," Applied Numerical Mathematics, vol. 61, no. 9, pp. 989-1000, 2011.

[15] L. Greengard, "Spectral integration and two-point boundary value problems," SIAM Journal on Numerical Analysis, vol. 28, no. 4, pp. 1071-1080, 1991. 